\title{
Unlicensed Health Care Personnel and Patient Outcomes
}

\author{
Thomas Bodenheimer, M.D. ${ }^{1,2}$ \\ ${ }^{1}$ Center for Excellence in Primary Care, University of California, San Francisco, CA, USA; ${ }^{2}$ Department of Family and Community Medicine, University \\ of California, San Francisco, CA, USA.
}

$\mathrm{J}$ Gen Intern Med 30(7):873-5

DOI: $10.1007 / \mathrm{s} 11606-015-3274-\mathrm{x}$

(c) Society of General Internal Medicine 2015

$I^{n}$ $\mathrm{n}$ this issue of JGIM, Balaban et al. report on a hospital readmission program within Cambridge Health Alliance, a high-performing safety-net health system in Massachusetts. ${ }^{1}$ In contrast to most interventions to reduce unnecessary readmissions, this one utilized unlicensed community health workers. For years, Cambridge Health Alliance (CHA) has employed community health workers whose job title is "patient navigator." Their role is "a bridge between patients and the health care system.” They help patients navigate through doctors, clinics, hospitals, insurance, support services, and other components of the health care system. The patient navigators are also trained in health coaching, utilizing motivational interviewing and action planning to assist patients in self-managing their chronic conditions. In addition, trilingual CHA patient navigators coordinate care, provide support, and help patients navigate the complexities of modern cancer treatment.

In this randomized controlled trial, the intervention consisted of patient navigators conducting one in-patient visit and three post-discharge phone calls to help patients arrange appointments, understand their care plans, improve medication adherence, and better self-manage their conditions. The results showed a reduction in readmissions for patients over the age of 60 , but not for those under 60 years of age. This result is important, because reducing readmissions is not only a cost outcome but is also a clinical quality and quality-of-life outcome; patients who are readmitted are in poor control of their disease, and admission to the hospital denotes poor quality of life.

What can we learn from this study? First, how do we understand the study results? Second, is there evidence that unlicensed health care personnel-for example, patient navigators - can improve care?

\section{KNOW YOUR PATIENTS}

Improvement programs are sometimes planned without understanding the patients for whom the improvement is targeted.

Published online March 28, 2015
The article by Balaban et al. reminds us that we need to know the patients. Why were the patient navigators successful for the over- 60 group and not for younger patients? It was not the age, but rather the nature of the patients' health problems, that explains the difference. Older patients (who tended to be Medicare beneficiaries) were more medically complex, while younger patients (many of whom were Medicaid recipients) had significantly more mental illness, substance abuse, and even dementia. Patient navigators had a difficult time contacting the younger patients, presumably due to the patients' psychosocial issues.

This finding mirrors the experience of organizations offering programs for patients with complex health care needs and high medical costs. ${ }^{2}$ Programs targeting Medicaid patients (similar to the under-60 group in Balaban et al.) need to be different from programs focusing on Medicare beneficiaries (Balaban's over-60 group). Medicaid-oriented complex care management teams generally include a social worker and nonlicensed outreach personnel (for example, patient navigators) who are trained to build relationships with patients who have difficulty trusting medical professionals. The programs focus on navigation, including teaching patients alternatives to calling 911. In contrast, teams for Medicare patients are often led by registered nurses and emphasize self-management support, in particular teaching patients and families what to do when yellow flags (symptom worsening) appear, to head off the red flags which often herald a hospital admission. As with the Balaban et al. study, programs aimed at Medicare patients have shown some success in reducing readmissions, ${ }^{3}$ while those for Medicaid patients have had more challenges to overcome. $^{2}$

\section{UNLICENSED HEALTH CARE PERSONNEL}

In ambulatory care, most unlicensed personnel are medical assistants and receptionists. Yet an emerging group of unlicensed health care workers is proliferating, including community health workers, patient navigators, health coaches, and peer educators. Categorizing these four groups is confusing, because they constitute a mix of job titles and job functions. "Patient navigator" is a job title within programs for cancer patients, but patient navigation is a function - helping patients to find their way through the maze of health care institutions. Similarly, while some unlicensed personnel carry the title of "health coach," health coaching is frequently a 
function-providing self-management support for patients with chronic conditions ${ }^{4}$ - often performed by medical assistants, trained patients (peer educators), and by such licensed personnel as nurses, social workers, health educators, or behavioral health providers. Community health workers - a job title - may be peers (coming from the same communities and having the same diseases as the patients they work with), and - as at Cambridge Health Alliance - their functions may include both patient navigation and health coaching.

The Balaban et al. study showed that for the older subgroup, patients working with community health workers had fewer readmissions than control patients. ${ }^{1}$ Does other evidence exist demonstrating that unlicensed personnel performing patient navigation or health coaching functions can improve health outcomes?

Patient navigation. Latina women with abnormal Pap smears who were assisted by patient navigators had fewer missed colposcopy visits, shorter times to colposcopy, and a reduction in the severity of cervical abnormalities seen on pathology specimens compared with a similar group of women without navigators. ${ }^{5}$ Similarly, Latina women with abnormal findings on mammography who were assisted by patient navigators had significantly shorter times to diagnosis than women in a control group without navigation. ${ }^{6}$

Health coaching. Of four randomized trials using community health workers as coaches for patients with diabetes, one found a significant reduction in $\mathrm{HbAlc}$ levels. In that study, community health workers led patient education classes, visited patients at home twice a month, and provided follow-up phone calls. ${ }^{7}$ Poor African-American children with asthma assisted by CHW "asthma coaches"-members of the same community - had significantly reduced asthma hospitalization rates compared with the randomized control group. ${ }^{8}$ In a randomized controlled trial of low-income patients with poorly controlled diabetes, patients with peer health coaches (other patients with diabetes) had significantly improved $\mathrm{HbA} 1 \mathrm{c}$ levels compared with controls. ${ }^{9}$ In a randomized controlled trial, patients with diabetes, hypertension, and/or hyperlipidemia who were linked with unlicensed personnel trained as health coaches had significantly improved $\mathrm{HgAlc}$ and LDLcholesterol after 1 year compared with non-coached patients. ${ }^{10}$

How does a medical practice or health system decide who should be responsible for which functions? Typically, functions are assigned according to the occupations or licensure of their clinician and non-clinician staff. Yet there is another way. Licensure is a poor indicator of success in assigning new roles such as patient navigation, self-management support, or care coordination among hospital, ambulatory care, and home.
How well people perform different functions may vary more with their personality, interests, and commitment than with their occupation or licensure. In a review of 44 articles on community health workers, the most commonly reported hiring criteria were interest in the subject material, willingness to learn, and compassion, rather than educational background or knowledge. ${ }^{11}$

We can move beyond licensure and consider which individuals are best suited to which functions. Some medical assistants can be trained to be excellent health coaches; others dislike coaching and do it poorly. Some RNs excel as coaches; others are more suited for acute care, are uninterested in coaching, and will flounder. Rather than ask "Should MAs or RNs provide health coaching?" primary care leaders might consider "Who is the best person to assume the coaching role?" Some community health workers may have more success than some physicians at encouraging patients to adhere to their medications. Some peer educators may be more effective than some RNs at inspiring patients to choose healthy behaviors. A research agenda might consider correlating hiring criteria with individual performance for a variety of health occupations.

Conventional thinking holds that licensed professionals are required to reduce hospital readmissions. ${ }^{3}$ But if personal qualities trump licensure for patient navigation and health coaching functions, it is not surprising that unlicensed community health workers at Cambridge Health Alliance were successful in assisting the over-60 patients to reduce readmissions.

Corresponding Author: Thomas Bodenheimer, M.D.; Department of Family and Community MedicineUniversity of California, Bldg 80-83, SF General Hospital 1001 Potrero Ave., San Francisco, CA 94110, USA (e-mail: TBodenheimer@fcm.ucsf.edu).

\section{REFERENCES}

1. Balaban RB, Galbraith AA, Burns ME, Vialle-Valentin CE, Larochelle MR, Ross-Degnan D. A patient navigator intervention to reduce hospital readmissions among high-risk safety-net patients: a randomized controlled trial. J Gen Intern Med. 2015 Jan 24.

2. Bodenheimer T. Strategies to reduce costs and improve care for highutilizing medicaid patients: Reflections on Pioneering programs. Center for Health Care Strategies, October 2013. www.chcs.org/resource/strategiesto-reduce-costs-and-improve-care-for-high-utilizing-medicaid-patients-reflections-on-pioneering-programs, accessed February 4, 2015.

3. Bodenheimer T, Berry-Millett R. Care Management for Patients with Complex Healthcare Needs, Robert Wood Johnson Foundation, 2009. www.rwjf.org/content/dam/farm/reports/issue_briefs/2009/rwjf49853/ subassets/rwjf49853_1, accessed February 4, 2015.

4. Ghorob A. Health coaching: teaching patients to fish. Family Practice Management. 2013;20(3):40-42.

5. Percac-Lima S, Benner CS, Lui R, Aldrich LS, Oo SA, Regan N, Chabner BA. The impact of a culturally tailored patient navigator program on cervical cancer prevention in latina women. $J$ Womens Health. 2013;22:426-431.

6. Ramirez AG, Pérez-Stable EJ, Penedo FJ, Talavera GA, Carrillo JE, Fernandez ME, et al. Navigating Latinas with breast screen abnormalities to diagnosis. Cancer. 2013;119:1298-1305.

7. Spencer MS, Rosland AM, Kieffer EC, Sinco BR, Valerio M, Palmisano G, et al. Effectiveness of a community health worker intervention among 
African- American and Latino adults with type 2 diabetes. Am J Public Health. 2011;101:2253-2260.

8. Fisher EB, Strunk RC, Highstein GR, Kelley-Sykes R, Tarr KL, Trinkaus K, Musick J. A randomized controlled evaluation of the effect of community health workers on hospitalization for asthma: the asthma coach. Arch Pediatr Adolesc Med. 2009; 163:225-232.

9. Thom DH, Ghorob A, Hessler D, DeVore D, Chen E, Bodenheimer T. Impact of peer health coaching on glycemic control in low-income patients with diabetes: a randomized controlled trial. Ann Fam Med. 2013;11:137-144.
10. Willard-Grace $\mathbf{R}$, Chen EH, Hessler D, DeVore $\mathbf{D}$, Prado $\mathbf{C}$, Bodenheimer T, Thom DH. Health coaching by medical assistants to improve control of diabetes, hypertension and hyperlipidemia for lowincome patients: a randomized controlled trial. Ann Fam Med, 2015;13:130-138.

11. O'Brien MJ, Squires AP, Bixby RA, Larson SC. Role development of community health workers. Am J Prev Med. 2009;37(6 Suppl 1):S262S269. 